\title{
Deutsch als Minderheitensprache
}

\author{
German as a Minority Language
}

\begin{abstract}
ZUSAMMENFASSUNG
Dieser Beitrag beschreibt Varietäten des Deutschen, die in exterritorialen deutschen Gemeinschaften gesprochen werden. Viele dieser Gruppen gehen auf Wanderbewegungen im Mittelalter oder in der frühen Neuzeit zurück und haben spezifische Varietäten entwickelt, die durch Dialektmischung und Sprachkontakt mit den Umgebungssprachen gekennzeichnet sind. Eine weitere Gruppe sind sogenannte „Grenzminderheiten“, exterritoriale Gemeinschaften, die nach dem Ersten Weltkrieg entstanden sind und an deutschsprachige Länder angrenzen. Der Artikel gibt zunächst einen historischen Überblick über die verschiedenen deutschsprachigen Minderheiten. Anschließend werden die unterschiedlichen soziolinguistischen Rahmenbedingungen der jeweiligen Sprachgemeinschaften angesprochen und anhand von Beispielen von Gemeinschaften mit unterschiedlichem soziolinguistischem und sprachlichem Hintergrund illustriert.

Schlüsselwörter: Deutschsprachige Minderheiten, Sprachinseln, Sprachkontakt, Dialektmischung
\end{abstract}

\section{ABSTRACT}

This contribution focusses on varieties of German which are spoken in extraterritorial German communities. Many of these groups go back to emigration in the Middle Ages or in Early Modern Times and have developed a specific koiné which is characterized by dialect merger and language contact with the surrounding languages. Another group are so-called "border minorities", extraterritorial communities that emerged after World War I and are bordering German-speaking countries. The article first provides a historical overview of the various German-speaking minorities. Then, the different sociolinguistic settings of the respective language communities are addressed and illustrated by examples of communities with a different sociolinguistic and linguistic background.

Keywords: German-speaking minorities, language enclaves, language contact, dialect merger

\section{Einführung}

Unter den verschiedenen Varietäten des Deutschen sind Sprachformen von besonderem Interesse, die außerhalb des geschlossenen deutschen Sprachraums gesprochen werden. Viele extraterritoriale Sprachgemeinschaften gehen auf Wan-

Claudia Maria Riehl, Institut für Deutsch als Fremdsprache, Ludwig-Maximilians-Universität München, Ludwigstraße 27, 80539 München, Phone: 00498921802116, riehl@daf.Imu.de, https://orcid. org/0000-0002-5591-2889

Rahel Beyer, Leibniz-Institut für Deutsche Sprache R5, 6-13, 68161 Mannheim, beyer@ids-mannheim. de, https://orcid.org/0000-0002-9306-8906 
derbewegungen im Mittelalter oder in der frühen Neuzeit zurück und werden in der germanistischen Forschung als „Sprachinseln“ bezeichnet, da sie über Jahrhunderte relativ isoliert vom Mutterland existierten, dort aber die Minderheitensprache bewahrten und ein kulturelles Eigenleben in ihrem relativ kleinen geschlossenen Siedlungsgebiet führten (vgl. Mattheier, 1996; Riehl, 2010). Weitere extraterritoriale Gemeinschaften sind das Resultat jüngerer Migrationsbewegungen im 19. Jh. Auch sie haben die deutsche Sprache in unterschiedlichen Formen über viele Generationen erhalten.

Eigene Spezifika und Dynamiken weisen außerdem die Varietäten auf, die zwar noch Teil des geschlossenen deutschen Sprachraums in Mitteleuropa sind, jedoch jenseits der Grenzen der deutschsprachigen Länder gesprochen werden, d.h. in direkten Nachbarstaaten, deren Mehrheitsbevölkerung eine andere sprachliche Varietät sprechen. Aufgrund der sprachlichen Verbundenheit zu einem sprachlichen Mutterland werden solche Minderheiten als „nationale Minderheiten“ (Eichinger, 2006, S. 2474-2475) bzw. aufgrund ihrer Grenzlage als „Grenzminderheiten“ bezeichnet (Pusch, 2010; Riehl, 2014). Im Folgenden wird zunächst ein historischer Überblick über die deutschen Sprachminderheiten gegeben, danach werden die unterschiedlichen soziolinguistischen Bedingungen der jeweiligen Sprachgemeinschaften erläutert. Davon ausgehend werden die verschiedenen Typen von Varietäten anhand einiger Beispielregionen vorgestellt.

\section{Historischer Überblick}

\subsection{Die Entstehung der deutschen Sprachinseln}

Die deutschen Sprachinseln lassen sich in zwei Gruppen unterteilen: die alten Sprachinseln aus der Zeit des Mittelalters, die vom 12. bis 14. Jh. besiedelt wurden, und neuere Siedlungen aus dem 17. bis 19. Jh. Aus der ersten Siedlungswelle im Mittelalter gibt es noch einige Restgruppen jenseits der Alpen, z.B. Walser Mundarten im Aosta-Tal und Reste der 7 und 13 Gemeinden in Oberitalien (die sog. ,Zimbern'). Die größeren Siedlungswellen gingen nach Osten. Förderer der Siedlungsbewegung waren Grundherren, die die fortschrittliche Agrartechnik und Wirtschaftsorganisation der deutschen Siedler für ihr Land in Anspruch nehmen wollten. Als Ausgleich boten sie dann entsprechende soziale Vorteile an (Gottas, 1995). Alte Sprachinseln finden sich vor allem im südöstlichen Mitteleuropa und in Südosteuropa, in Ungarn, der Slowakei und Rumänien (Siebenbürgen). Die zweite Siedlungswelle setzte Ende des 17. Jhs. ein und erstreckte sich teilweise bis in die Mitte des 19. Jhs. Viele der neuzeitlichen Siedlungen wurden nach Beseitigung der Türkenherrschaft in Südost- und Osteuropa gegründet (z.B. in Ungarn, NordRumänien, im Banat und der Batschka). In diese Zeit fällt auch die Kolonisation deutschsprachiger Gebiete in Russland; es entstanden deutschsprachige Dörfer 
an der Wolga, in der Gegend um St. Petersburg und am Schwarzen Meer ${ }^{1}$. Gleichzeitig begann auch die Übersiedlung in überseeische Gebiete, nach Nordund Südamerika und nach Australien (vgl. Riehl, 2010, S. 81-82).

In den meisten Fällen waren wirtschaftliche Gründe ausschlaggebend für die Auswanderungen, aber es gab auch religiöse Motive: So siedelte etwa die ursprünglich aus der Schweiz stammende Sekte der Amischen (Amish people) zunächst im Pfälzer Raum, als aber auch hier der Druck auf diese Religionsgemeinschaft zu groß wurde, ließen sie sich ebenfalls in den USA nieder, und zwar in Pennsylvania (ab 1730). Eine noch weitere Wanderbewegung machten einige Mennonitengruppen mit: Diese stammen aus den Niederlanden, siedelten im Danziger Werder, dann im Schwarzmeergebiet und im weiteren Russischen Reich und wanderten nach Verlust ihrer Privilegien Ende des 19. Jh. und nach Verfolgungen in der Frühzeit der Sowjetunion nach Kanada aus, ein Drittel zog nach Mexiko sowie nach Südamerika weiter (vgl. Siemens, 2018). Diese religiösen Gruppen haben durch ihre konservative Haltung und Isolation einen sehr alten Sprachstand bewahrt und verwenden teilweise auch eine eigene Schriftsprache für ihre religiösen Schriften (Siemens, 2018).

Während viele Sprachinseln in Osteuropa im 19. Jh. Dialektenklaven darstellten, deren Sprecher minimalen Kontakt mit der Standardvarietät des Deutschen hatten, hat sich das im 20. Jh. nicht nur durch den Kontakt mit der genetisch verwandten Standardsprache des Deutschen, die in der Schule gelehrt wurde, verändert, sondern auch durch den zunehmenden Kontakt mit den Umgebungssprachen. Besonders nach den einschneidenden Veränderungen nach dem Zweiten Weltkrieg (Vertreibung oder Umsiedlung der deutschen Bevölkerung, Verbot der deutschen Sprache) kam es zu einer starken Verlagerung in der Dominanz der Sprachen, die sich auch auf die deutschen Varietäten auswirkt (Riehl, 2019).

Im Gegensatz zu den Gebieten in Osteuropa sind die deutschsprachigen Gemeinschaften in Übersee viel jünger und umfassten oft zu wenige Generationen, damit sich neue Sprachvarietäten herausbilden konnten (vgl. Boas, 2009, S. 293). Ausnahmen bilden allerdings die religiösen Gruppen (Amische, Mennoniten etc.), die aufgrund ihrer religiösen Gruppenbildung einen Sonderstatus bilden und sich häufig von der Mehrheitsgesellschaft isolieren (vgl. hierzu Siemens, 2018)2.

Ein weiterer Unterschied, der im Falle der überseeischen Besiedlung eine Rolle spielt, ist die Besiedlung von Städten und der ständige Nachzug von deutschen

1 Die neue Siedlungsbewegung unterscheidet sich von der mittelalterlichen Besiedlung in einigen wesentlichen Punkten (Gottas 1995, S. 19): Es handelt sich hier um eine „von oben gelenkte Bevölkerungsbewegung und um planmäßige Siedlungspolitik“. Diese wurde nicht nur von Österreich-Ungarn, sondern auch von Preußen betrieben und später von Katharina II. aufgegriffen.

2 Bei den mennonitischen Gruppen kommt hinzu, dass diese oft bereits aus anderen Sprachenklaven, etwa Ukraine oder Russland, auf den amerikanischen Kontinent auswanderten (vgl. Kaufmann, 2004; Siemens, 2018). 
Auswanderern aus dem Mutterland besonders nach dem Zweiten Weltkrieg. Das betrifft vor allem die deutschsprachigen Gebiete in den USA (z.B. TexasGerman) und Australien. Diese Tatsache führte einerseits zu einer ständigen Spracherneuerung, kann aber auch eine Erklärung für die fehlende Ausbildung von eigenen Ausgleichsvarietäten (im Sinne einer Koiné) liefern. Außerdem werden diese Gruppen ,nicht mehr durch eine insulare Abgeschiedenheit, sondern vielmehr durch Zwei- und Mehrsprachigkeit und Sprachen- bzw. Kulturenkontakte bestimmt" (Földes, 2006, S. 330).

Ist schon für die Zeit der Entstehung der Begriff Sprachinsel in seiner ursprünglichen dialektologisch geprägten Definition für eine Reihe von Siedlungen in Nordund Südamerika fraglich, die keine eigenen Dialekte oder Ausgleichsvarietäten ausgebildet haben, so wird sie umso problematischer für Gemeinschaften, die bereits in Auflösung begriffen sind: Das betrifft die Gemeinschaften in Chile und Argentinien (vgl. Rosenberg, 2018) ebenso wie die deutschsprachigen Gruppen in Texas, im Mittelwesten der USA sowie in Australien.

\subsection{Historischer Überblick über die Grenzminderheiten}

Mit dem Ende des Mittelalters waren die Sprachgrenzen des deutschen Sprachgebiets in Europa im Wesentlichen etabliert, wobei es v.a. im Norden und Osten, wie z.B. in Schleswig, den böhmischen Ländern und verschiedenen Gebieten Polens, auch zu einer Überlagerung der Sprachgebiete bzw. zu einem Zusammenleben deutsch- und anderssprachiger Bevölkerung kam. Die ab der Neuzeit gezogenen politisch-administrativen Grenzen durchtrennten nun mitunter die etablierten Sprachgebiete, z.B. wenn Staaten andere, „natürliche“ Grenzen (Gebirgszüge, Flüsse) als Staatsgrenzen anvisierten (Pusch, 2010, S. 380). Andererseits bildete gerade die Existenz deutschsprachiger Bevölkerung in einem Territorium häufig den Anlass für dessen Beanspruchung durch Preußen bzw. das Deutsche Reich. In der Konsequenz bedeutete dies ein häufiges Wechseln der staatlichen Zugehörigkeit, teils durch diplomatische Verhandlungen, meistens jedoch durch Annexion und Rückeroberung. So ging zunächst etwa nach dem Westfälischen Frieden (1648) ein größerer Teil des damaligen elsässischen Gebiets und 1766 das Herzogtum Lothringen per Erbregelung an Frankreich über. Ende des 18. Jahrhunderts und im Laufe des 19. Jhs. wurden dann nach und nach einige Gebiete Polens, Eupen-Malmedy-Sankt Vith (1815), Schleswig (1864) sowie das Elsass und ein Teil Lothringens (wieder) an Preußen bzw. das spätere Deutsche Reich angegliedert. Relativ stabil waren die Verhältnisse in Südtirol. Als Teil von Gesamttirol war es zunächst bis 1806 Teil des Heiligen Römischen Reichs Deutscher Nation, danach Österreichs (vgl. z.B. Ammon, 2007, 105-109). Allen heutigen Grenzminderheiten ist also gemein, dass sie am Anfang des 20. Jahrhunderts alle auch politisch mit dem geschlossenen deutschen Sprachgebiet vereint waren. 
Das Ende des Ersten Weltkriegs bzw. die Niederlage des Deutschen Reichs und Österreich-Ungarns bildet einen Wendepunkt. Die darauffolgenden neuen Grenzziehungen wurden v.a. durch den Versailler Vertrag geregelt, der größtenteils eine Rückgliederung der im 19. Jahrhundert erworbenen Gebiete bestimmte. Der nördliche Teil Schleswigs entschied sich im Rahmen einer Volksabstimmung mit großer Mehrheit für einen Anschluss an Dänemark. Das überwiegend deutschsprachige Südtirol wurde im November 1918 von Italien besetzt und mit dem Vertrag von Saint-Germain 1919 endgültig an den italienischen Staat angegliedert.

In der Zeit zwischen den beiden Weltkriegen wurde die deutsche Sprache in sämtlichen Grenzterritorien aus Öffentlichkeit, Schule und Kirche erheblich zurückgedrängt und die jeweilige Mehrheitssprache offensiv gefördert. Dabei waren die Zustände in einigen Staaten recht prekär (vgl. z.B. die Majorisierung und Assimilationsbestrebungen in Südtirol). Unter der nationalsozialistischen Herrschaft wurde andererseits 1938 das Münchner Abkommen errungen, das eine Abtretung aller tschechoslowakischen Gebiete mit einer mehr als 50-prozentigen deutschen Bevölkerung an das Deutsche Reich vorsah (vgl. z.B. Born \& Dickgießer, 1989, S. 220).

Mit dem Zweiten Weltkrieg und dem Streben der NS-Regierung, ein Großdeutsches Reich zu errichten, kam es zu einem erneuten Umbruch. So kamen die deutschsprachigen Grenzminderheiten wieder unter die politische Führung des Deutschen Reichs. Damit einher ging in der Regel auch eine radikale Politik der Germanisierung - mit tiefgreifenden Folgen für die Bevölkerung.

Nach der Niederlage des Deutschen Reiches wurden die Staatsgrenzen von 1919 teils wiederhergestellt. Während die Deutschsprachigen in Dänemark und Belgien relativ konfliktfrei eingegliedert wurden (bereits 1955 wurde die Minderheit in Dänemark etwa mit der Bonn-Kopenhagener Erklärung offiziell anerkannt), erlebten sie in Frankreich Stigmatisierung und Anfeindung, in Polen und Tschechien Vertreibung und Assimilierungsdruck. Von den Südtirolern wurde eine Rückkehr zu Österreich angestrebt. Ihr 1946 gestellter Antrag zur Selbstbestimmung wurde jedoch von den Alliierten abgelehnt (vgl. u.a. Beyer \& Plewnia, 2021).

Während Entstehung (politische Abtrennung vom sprachlichen Gesamtgebiet durch Grenzziehung) und die jüngere Geschichte der Grenzminderheiten in den Grundzügen ähnlich sind, gibt es auch Merkmale, die in den jeweiligen Gebieten unterschiedlich ausgeprägt sind. So waren die angrenzenden Sprachminderheiten in Tschechien und Polen lange Zeit aufgrund des Eisernen Vorhangs isoliert von den Ländern mit deutscher Mehrheitssprache (vgl. Riehl, 2014, S. 66) - anders als jene im Westen und Süden. Auch die räumlichen Gegebenheiten unterscheiden sich: So leben manche Minderheiten verstreut unter der Mehrheitsbevölkerung (z.B. in Nordschleswig). Andere wiederum lassen sich klar einem Territorium zuordnen, in dem sie auch eine regionale Mehrheit darstellen (z.B. in Ostbelgien). 
Angesichts der teilweise offiziellen Gleichstellung scheint der - je nach Definition - eine Marginalisation implizierende Begriff „Minderheit“ nicht für alle betroffenen Gebiete passend (Riehl, 2014, S. 66).

\section{Soziolinguistische Bedingungen}

Aus den Ausführungen in Kap. 2 ist erkennbar, dass in den deutschen Minderheitengebieten je nach Lage und Alter der Siedlung ganz unterschiedliche Sprachkontaktkonstellationen vorhanden sind.

Aus gegenwärtiger Sicht ist der Erhalt der deutschen Sprache, die Ausprägung eines Varietätenspektrums (Dialekt - Regionalsprache - Standard) und der Einfluss der Umgebungssprachen in den jeweiligen Gebieten maßgeblich durch den jeweiligen Status der deutschen Sprache geprägt: Dieser reicht von der vollständigen Gleichberechtigung der deutschen Sprache mit der Landessprache (Beispiel Südtirol) über die Anerkennung des Minderheitenstatus' (Beispiel Ungarn) bis zur völligen Nichtbeachtung (Beispiel Australien). Ein zentraler Aspekt ist dabei der Zugang zur Schriftlichkeit, z.B. über das Vorhandensein des Deutschen als Schulsprache oder religiös bedingt, etwa wenn in bestimmten konfessionellen Gruppen (Protestanten, Mennoniten) ein traditionell höherer Zugang zu Bildung und darüber auch zur Schriftlichkeit besteht (vgl. Riehl, 2014, S. 143-144).

Zur Illustration sollen die unterschiedlichen Typen von Minderheitsregionen im Folgenden anhand von Beispielregionen beschrieben werden.

\subsection{Pluriglossie und Varietätenspektrum: Beispiel Südtirol}

Wie in 2.2 dargelegt, teilen die Grenzminderheiten Teilaspekte der Entstehungsbedingungen und jüngeren Geschichte, zeigen aber in anderen Merkmalen deutliche Unterschiede. Dies betrifft auch die Vitalität und ,Ausgebautheit‘ des Deutschen. Südtirol ist einer der wenigen Fälle einer deutschen Sprachminderheit, wo Deutsch nicht nur in allen Domänen des Lebens gebraucht wird, sondern auch die Sprache der Alphabetisierung in der Schule ist, so dass dort eine komplett ausgebaute Sprache mit (bairischen) Dialekten und überdachender Standardsprache vorliegt. Nach einigen teils gewaltsamen Auseinandersetzungen als Reaktion auf die Fremdherrschaft trat im Jahr 1972 das zweite Autonomiestatut in Kraft, das Südtirol als „Autonome Provinz Bozen“ mit einer Reihe von Minderheitenrechten ausstattet (Dekret des Präsidenten der Republik vom 31. August 1972, Nr. 670), wie u.a. die offizielle Zweisprachigkeit. Deutsch ist - zumindest regional - dem Italienischen als Amtssprache gleichgestellt und wird in allen Bereichen der Verwaltung, bei Gericht und auch als Schulsprache verwendet. Unter diesen Bedingungen zeigt das Deutsche in Südtirol eine hohe Vitalität mit einer Vielzahl von Varianten bzw. Varietäten (Rabanus, 2018), „,deren Konventionalisierungsgrad unklar ist“" (Glück, Leonardi, \& Riehl, 2019, S. 256). Die Existenz einer 
deutschen Umgangssprache etwa ist bislang nicht eindeutig belegt, auch wenn es erste Hinweise in diese Richtung gibt (vgl. S. 256-258). Einer Einordnung des Verhältnisses der deutschen Varietäten als mediale Diglossie widerspricht einmal die schriftliche Verwendung von Dialekten zumindest in digitaler Kommunikation sowie die Verwendung der (regionalen) Standardsprache in mündlichen politischen TV-Debatten (Rabanus, 2018). Der deutsche Standard in Südtirol weist einige Besonderheiten auf, die häufig (aber nicht gänzlich) aus dem Dialekt stammen: Etwa im Bereich Phonetik (z.B. die Stimmlosigkeit wortinitialer $s$ oder die Lenisierung wortinitialer $p$ und $t$; Glück et al., 2019, S. 259), Morphosyntax (z.B. die Präpositionalphrasen am statt auf dem im Funktionsverbgefüge am Laufenden bleiben; Glück \& Leonardi, 2019, S. 457) und Lexik (z.B. Griffelschachtel für Federmäppchen oder Notspur für Standstreifen; Abfalterer, 2007, S. 108, 128). Hinzu kommen Entlehnungen aus dem Italienischen in begrenztem Umfang (von unassimilierten Lehnwörtern wie Carabiniere [Militärpolizist] bis hin zu Lehnübersetzungen wie Identitätskarte [Personalausweis] < ital. 'carta d'identità'). Ab der 1. Klasse der Grundschule wird an Schulen mit deutscher Unterrichtssprache außerdem Italienisch als Zweitsprache unterrichtet, so dass nativ deutschsprachige Südtiroler zumindest sukzessiv bilingual sind.

\subsection{Autochthone Dialekte mit Sprachkontakterscheinungen: Beispiel Elsass und Ostlothringen}

Auch wenn Grenzminderheiten sich in geographischer Kontaktstellung mit dem (oder einem) Sprachkernland befinden, ist nicht immer auch sozialpsychologisch eine Verbindung gegeben, so z.B. im Elsass und in Ost-Lothringen, wo die germanischen Varietäten in ungünstigen Bedingungen stehen. Mit der Stigmatisierung der mosel- und rheinfränkischen sowie alemannischen Dialekte als Sprache des Feindes und des Standarddeutschen als Nazisprache unmittelbar nach dem Krieg gingen dort eine tiefe Identitätskrise der Sprecher und ein starker Rückgang der transgenerationellen Weitergabe einher (Dorner, 2012). Als einzige Möglichkeit, an der für die Identität wichtigen Sprache der Nähe, den Dialekten, festzuhalten, erschien eine konzeptionelle Trennung vom (Standard-)Deutschen. Diese Einstellung verbreitet sich seit den 1970er Jahren immer mehr (Beyer \& Fehlen, 2019, S. 144). Unter „Deutsch“ wird heute im gesellschaftlichen Diskurs meist die deutsche Standardsprache des Nachbarlandes, der Bundesrepublik Deutschland, verstanden (Huck \& Erhart, 2019, S. 156). Die autochthonen Dialekte sind in diesem Sinne „dachlose Außenmundarten“ (Kloss, 1978) bzw. werden von manchen als autonome Sprache betrachtet (Elsässisch und Lothringer Platt bzw. Fränkisch).

Weder Deutsch noch die entsprechenden Dialekte haben in Frankreich eine stabile rechtliche Absicherung. Beide sind einzig im Bereich von Erziehung und Schule anerkannt (Ammon, 2007, S. 111). 1999 hat Frankreich zwar die Europäische Charta der Regional- oder Minderheitensprachen unterzeichnet; 
alle Ratifizierungsversuche sind bis heute jedoch gescheitert. Bedingt durch das fehlende deutsche Dach und den intensiven Kontakt mit dem Französischen gibt es in den Dialekten viele Entlehnungen im Bereich der Lexik (u.a. Boll [Schüssel/ Schale] < frz. ,bol'; Rispail, Haas-Heckel, \& Atamaniuk, 2012, S. 249; Verben auf-iere wie z.B. schànschiere < frz. ,changer', riskiere < frz. 'risquer'; Koehler, 2017, S. 65) und der Interaktion und Pragmatik (z.B. Partikeln wie enfin, bon, allez); außerdem finden sich Lehnübersetzungen (z.B. No hàw i e Johr Recht gemàcht [Dann hab ich ein Jahr Recht studiert] < frz. ,j'ai fait un an de droit') sowie Code-Switching (Koehler, 2017, S. 66-69).

\subsection{Mischdialekte und Koinés: Beispiel Wolgadeutsch}

Im Gegensatz zu den Grenzminderheiten, in denen die autochthonen Dialekte erhalten wurden und, wie in 3.2 beschrieben, sich im Kontakt mit der Umgebungssprache wandelten, finden wir in den sog. Sprachinseln des Deutschen andere Verhältnisse vor: Da hier in der Regel Siedler aus verschiedenen Gegenden des deutschen Sprachraums aufeinandertrafen, entstanden dort sog. Mischmundarten. Bei diesen Mischungsprozessen werden in der Regel im Verhältnis zur Standardsprache oder einer Kontaktvarietät besonders auffallende Besonderheiten der Dialekte (die primären Merkmale) gegenüber den primären Merkmalen dieser Varietät aufgegeben, selbst wenn sie von einer Mehrheit vertreten sind, während weniger auffallende (,sekundäre“) Merkmale im Allgemeinen auch in der Mischmundart auftreten (Protze, 1995, S. 58). Allerdings kann man Entwicklungen beobachten, wonach Merkmale, die sich besonders stark vom Standard unterscheiden, ausgewählt werden, vermutlich weil sie als authentischer angesehen werden (vgl. Rosenberg, 2005).

In einigen Regionen entstand auch eine eigene Verkehrsvarietät, eine Koiné. Ein besonders prominentes Beispiel ist hier das Hunsrückische in Südbrasilien (vgl. Altenhofen, 2016). Aber auch das sog. Wolgadeutsche zeigt deutliche Ansätze einer Koiné: Hier haben sich die wolgadeutschen Mischmundarten, die auf rheinpfälzischen, hessischen und ostmitteldeutschen Stammmundarten beruhten, nach der Vertreibung der deutschen Siedler im Zuge des Ersten Weltkriegs in andere Regionen der ehemaligen UdSSR einem weiteren Angleichungsprozess unterzogen. Dabei haben sich entweder die westmitteldeutsche Basis oder standardnähere Varianten durchgesetzt. Die mhd. Diphthonge ou und ei werden in den meisten Fällen wie im Standarddeutschen als $a u$ bzw. ai artikuliert: laufe [laufen], saif [Seife] und nicht mehr wie im rheinpfälzischen Dialekt als laafe, seef, oder wie im Hessischen als laafe, saaf (Berend \& Riehl, 2008, S. 38).

Im phonetisch-phonologischen Bereich fallen weiter Spirantisierung von Plosiven im Inlaut (gleche [gelegen], bliewe [geblieben]) und die Assimilation von $d$ nach Nasal (verstanne [verstanden]), $e$ - und $n$-Apokope sowie Entrundung auf. Phonologische Prozesse können auch zu morphologischen Wandelprozessen bei- 
tragen: So wird etwa der Kasussynkretismus durch Verwendung eines Einheitsartikels de (de Kinner, mit de Frau) und Abbau des n-Flexivs (mit de Kinner) (Apokopierung von auslautenden Konsonanten) unterstützt (vgl. Rosenberg, 2016).

An dialektaler Lexik treten bei allen Sprechern die folgenden Lexeme auf: schwach [schlecht], bang sein [Angst haben], alleinig [allein], Mannsleit [Männer], Weibsleit [Frauen], sellmal [damals], springen [laufen], verzählen [sprechen], Bud [Geschäft], sowie die Gradpartikel arch [sehr] (Berend \& Riehl, 2008, S. 38).

\subsection{Standardnahe Varietäten: Beispiel Deutsch in Namibia}

Während die in 3.3. beschriebenen Sprachinseln bereits auf eine über 200jährige Geschichte zurückblicken, sind die Siedlungen in Übersee in der Regel wesentlich jünger: Das hat zur Folge, dass sich dort oft keine eigenen Dialekte oder Mischmundarten herausgebildet haben, sondern eine standardnahe Varietät gesprochen wird, die regionaldeutsche Züge trägt und einige Sprachkontakterscheinungen aus der Umgebungssprache zeigt.

Ein prominentes Beispiel für eine derartige Varietät ist das Deutsche in Namibia, das noch von ca. 20.000 Sprechern gesprochen wird. Im gesprochenen Medium hat sich innerhalb der deutschen Sprachgruppe eine Varietät herausgebildet, die im Allgemeinen mit „Südwester-Deutsch“ bezeichnet wird (vgl. Gretschel, 1984). Neuerdings wird auch der Begriff „Namdeutsch“ (Wiese \& Bracke, 2021; Zimmer, 2019) verwendet. Diese Varietät zeigt eine wesentlich an einem norddeutschen Standard orientierte Lautung, was sich v.a. durch die Tatsache erklären lässt, dass die Siedler aus allen Teilen des deutschsprachigen Raums kommen. Damit unterscheidet sich dieses Gebiet maßgeblich von den dialektal geprägten Sprachinseln. Darüber hinaus kommt hinzu, dass der Anteil der aus Ostpreußen und Nordrhein-Westfalen zugewanderten Namibier, die teilweise bereits zur Zeit der Einwanderung keinen Dialekt mehr sprachen, wesentlich höher ist als der Anteil der Einwanderer aus dem südlichen Teil Deutschlands.

$\mathrm{Zu}$ den Charakteristika des Deutschen in Namibia zählen v.a. Entlehnungen aus dem Afrikaansen, und zwar neben Substantiven auch Adjektive (moii [schön, hübsch], lelik [häßlich]), Verben (morschen < om te mors [mantschen, pantschen, schmieren, vergeuden], frotten $<$ om te frot [verderben]) und Diskursmarker und Gradpartikel (wrachtach $<$ wrachtig [wirklich], mos $<$ mos [nur]). Daneben kommen auch Entlehungen aus dem Englischen vor (orreit $<$ alright). Sehr häufig sind auch Bedeutungsübernahmen, die sowohl vom Afrikaansen, als auch vom Englischen beeinflusst sein können: Manchmal ist diese Straße sehr beschäftigt. (afr. ,besig' [beschäftigt, tätig, überlaufen]); Hier ist noch ein Happie, dann bist du klar. (afr. ,klaar' [fertig, bereit]) (vgl. Dück, 2018; Riehl, 2014, S. 99-103).

Ebenfalls durch die Kontaktsprache beeinflusst, sind Erscheinungen in der Syntax. Hier wird bisweilen die Argumentstruktur des Afrikaansen ins Deutsche übertragen: Sie muß das Haus aufpassen, afr. ,sy moet die huis oppas' oder Ich 
muß für Katzenkost gucken, afr. ,om te kyk vir' (Riehl, 2014, S. 36). Daneben gibt es sprachinterne Wandelerscheinungen - etwa die Grammatikalisierung nichtkanonischer Formen -, die nicht auf den Sprachkontakt zurückzuführen sind (vgl. Wiese \& Bracke, 2021).

\subsection{Reliktvarietäten: Beispiel Barossadeutsch}

Eine besondere Gruppe innerhalb der Sprachinselvarietäten stellen sog. Reliktvarietäten dar. Darunter versteht man Sprachvarietäten, die folgende Kriterien aufweisen (vgl. Riehl, 2016, S. 262):

- Die Minderheitensprache wird nur von einer kleinen Anzahl von Sprechern der ältesten Generation verwendet.

- Die Sprecher erwarben ein unvollständiges System einer bereits vereinfachten Varietät.

- Die Sprecher erhielten variablen Input.

- Die Sprecher erwarben kein Schriftsystem der L1-Varietät.

- Die Sprecher leben isoliert von anderen Sprechern der gleichen L1.

Daher weisen Reliktvarietäten viele Anzeichen von Sprachabbau auf. Ein Beispiel dafür ist das sog. Barossadeutsche in Südaustralien. In dieser Sprachgemeinschaft konnte mit dem Beginn des Zweiten Weltkrieges Deutsch nicht mehr im öffentlichen Kontext verwendet werden und auch der private Gebrauch wurde eingeschränkt. Dies führte dazu, dass die Verwendungskontexte immer mehr abnahmen und sich ganz auf die Familie und Gespräche mit der älteren Generation beschränkten (vgl. Riehl, 2016). Heute leben nur noch ca. 40 Sprecher des Barossadeutschen, die alle schon über 80 Jahre alt sind.

Ähnlich wie das Deutsche in Namibia basiert auch Barossadeutsch auf einer regionalen Umgangssprache (Riehl, 2018, S. 19). Diese ist in der Lautung stark vom Englischen beeinflusst: Beispielsweise werden ungerundete Diphthonge der ursprünglich ostmitteldeutsch geprägten Umgangssprache wie /EI/ an Stelle von standarddt. /e:/ in Wörtern wie lesen sowie der Diphthong / $\varepsilon v /$ für standarddt. /o:/ in $g r o \beta$ verwendet. Durch den Sprachkontakt mit dem Englischen werden nun diese Diphthonge des deutschen Dialekts zu / $/ \mathrm{I} /$ wie in engl. wine oder $/ \Lambda \mathrm{v} /$ wie in engl. cow. Zudem verwenden die Barossadeutschen häufig alveolares [I] in Wörtern wie Regen (Clyne, 2003, S. 116).

Das Barossadeutsche enthält sehr viele aus dem Englischen entlehnte Lexeme, die überwiegend aus den drei wichtigsten Bereichen Farm, Obstanbau und Weinbau stammen: vgl. die Roode [road], die Kricke [creek[, der Rai [rye], der/ die Fenz [fence], die Päddock [paddock] die Laine [railway line] die Raasberi [raspberry], das Taunschipp [township] (vgl. Riehl, 2018, S. 21). Neben den lexikalischen Übernahmen gibt es auch zahlreiche semantische Übernahmen in Form von Lehnübersetzungen, z.B. fehlen [durchfallen bei der Prüfung], engl. ,to fail', Tee oder Teei [Abendbrot], uffgebracht [aufgezogen], engl. ,brought up'. 
Im Bereich der Syntax und Morphologie zeigen sich eine Reihe von Vereinfachungsprozessen: So ist bereits der Dativ vollständig abgebaut und nur noch in wenigen häufigen Verbindungen, etwa mit Verwandtschaftsbezeichungen (mit meiner Mutter) oder in festen Wendungen wie (zur Kirche, zur Schule) erhalten. Auch im Bereich der Personalpronomina wird die alte Dativ- und Akkusativ-Distinktion durch einen obliquen Kasus ersetzt (vgl. Riehl, 2016).

Ein typischer Restrukturierungsprozess im Rahmen des Sprachabbaus zeigt sich in der Präteritumsbildung: Hier wird öfter die tun-Periphrase, die ursprünglich die Funktion einer gewohnheitsmäßigen Umschreibung hat, genutzt, um Präteritum im Allgemeinen auszudrücken (auch bei einmaligen Handlungen, vgl. Und es war n police-Mann. Der tat alle die Flinten ... einnehm, Riehl, 2018, S. 24).

\section{Zusammenfassung: Entwicklungstendenzen}

Unser kurzer Überblick hat gezeigt, dass es sich bei den Minderheitenvarietäten des Deutschen um ein sehr breites Spektrum von Sprachvarietäten handelt, die von einem völlig ausgebauten polyglossischen System mit einem regionalen Standard und einem Dialektspektrum (Beispiel Südtirol) über dachlose Dialekte (Beispiel Elsass-Lothringen) zu Sprachminderheiten mit einer gemeinsam ausgebildeten Koiné (Beispiel Wolgadeutsch) reicht. Viele Sprachinselminderheiten erleiden allerdings das Schicksal des Sprachabbaus, so dass man hier von Reliktvarietäten sprechen muss (Beispiel Barossadeutsch). Prozesse wie etwa der Kasusabbau lassen sich aber in allen Varietäten feststellen, hier nur in unterschiedlichen Stadien: Hierbei handelt es sich um eine typologische Entwicklung, die durch den Sprachkontakt und die Isolierung vom Sprachkernland allenfalls beschleunigt wird, indem interner und externer Sprachwandel interagieren (Rosenberg, 2016). Andere Phänomene, wie etwa die Lexik und Semantik sind von der jeweiligen Kontaktsprache beeinflusst.

Was die Auswirkungen des Sprachkontakts angeht, so kann man von einem Kontinuum sprechen zwischen Sprachgemeinschaften, die nur wenige Sprachkontakterscheinungen im Bereich der Lexik und Semantik zeigen (wie Südtirol oder Namibia) über Sprachminderheiten, die neben lexikalischen auch morphosyntaktische und pragmatische Einflüsse aufweisen (wie Elsass und Ostlothringen) bis hin zu den Gemeinschaften, die neben den direkten Sprachkontakterscheinungen auch Simplifizierungs- und Restrukturierungsprozesse zeigen (wie das Barossadeutsche).

Der jeweilige „Zustand“ der Sprache ist maßgeblich von soziolinguistischen Bedingungen, v.a. dem Institutionalisierungsgrad der Sprache, abhängig. Dort, wo das Deutsche auch als Schriftsprache verwendet wird, ist der Einfluss des Sprachkontakts am geringsten. Allerdings ist in letzter Zeit auch eine neue Dynamik zu beobachten: So bieten etwa die neuen Medien und Kontakte über verschiedene Formen von social media mit Verwandten oder Freunden 
in den deutschsprachigen Ländern neue Möglichkeiten der Vernetzung über Grenzen hinweg, die die Verwendung der deutschen Sprache zusätzlich fördern (vgl. Riehl, 2016).

\section{References}

Abfalterer, H. (2007). Der Südtiroler Sonderwortschatz aus plurizentrischer Sicht. Lexikalischsemantische Besonderheiten im Standarddeutsch Südtirols. Innsbruck: Innsbruck University Press.

Altenhofen, C. V. (2016). Standard und Substandard bei den Hunsrückern in Brasilien: Variation und Dachsprachenwechsel des Deutschen im Kontakt mit dem Portugiesischen. In A. Lenz (Ed.), German Abroad. Perspektiven der Variationslinguistik, Sprachkontaktund Mehrsprachigkeitsforschung (pp. 103-129). Wien: V\&R unipress.

Ammon, U. (2007). Deutschsprachige Minderheiten in Europa im Vergleich zum Elsass. In J. Darquennes (Ed.), Kontaktlinguistik und Sprachminderheiten (pp. 103-116). St. Augustin: Asgard.

Berend, N., \& Riehl, C. M. (2008). Russland. In L. M. Eichinger, A. Plewnia, \& C. M. Riehl (Eds.), Handbuch der deutschen Sprachminderheiten in Mittel- und Osteuropa (pp. 17-58). Tübingen: Narr.

Beyer, R., \& Fehlen, F. (2019). Der germanophone Teil Lothringens. In R. Beyer, \& A. Plewnia (Eds.), Handbuch des Deutschen in West- und Mitteleuropa. Sprachminderheiten und Mehrsprachigkeitskonstellationen (pp. 106-154). Tübingen: Narr.

Beyer, R., \& Plewnia, A. (Eds.) (2019). Handbuch des Deutschen in West- und Mitteleuropa. Sprachminderheiten und Mehrsprachigkeitskonstellationen. Tübingen: Narr.

Boas, H. C. (2009). The Life and Death of Texas German. Durham: Duke University Press.

Born, J., \& Dickgießer, S. (1989). Deutschsprachige Minderheiten. Ein Überblick über den Stand der Forschung für 27 Länder. Mannheim: Institut für Deutsche Sprache.

Clyne, M. (2003). Dynamics of Language Contact. English and Immigrant Languages. Cambridge: Cambridge University Press.

Dekret des Präsidenten der Republik vom 31. August 1972, Nr. 670. Genehmigung des vereinheitlichten Textes der Verfassungsgesetze, die das Sonderstatut für Trentino-Südtirol betreffen. Retrieved March 1, 2018, from http://lexbrowser.provinz.bz.it/doc/de/dpr-1972670/dekret_des_pr_sidenten_der_republik_vom_31_august_1972_nr_670.aspx?view=1.

Dorner, D. (2012). Social actors and the language policy and planning process: A case study from German-speaking Lorraine (France). In P. Studer, \& I. Werlen (Eds.), Linguistic Diversity in Europe. Current Trends and Discourses (pp. 157-175). Berlin, Boston: De Gruyter.

Dück, K. (2018). Namibia. In A. Plewnia, \& C. M. Riehl (Eds.), Handbuch der deutschen Sprachminderheiten in Übersee (pp. 109-130). Tübingen: Narr.

Eichinger, L. M. (2006). Soziolinguistik und Sprachminderheiten. In U. Ammon, N. Dittmar, K. J. Mattheier, \& P. Trudgill (Eds.), Soziolinguistik. Ein internationales Handbuch zur Wissenschaft von Sprache und Gesellschaft: Vol. 3 (pp. 2473-2484). Berlin, New York: De Gruyter.

Földes, C. (2006). „Sprachinsel“-Paradigma auf dem Prüfstand. Konzept, Terminologie und Forschungsmethodologie. Zeitschrift für Deutsche Philologie, 125, 321-341.

Glück, A., \& Leonardi, M. M. V. (2019). Zur Verwendung von Präpositionen in Texten und Diskursen von Südtiroler Maturanten. In S. Kürschner, M. Habermann, \& P. O. Müller (Eds.), Methodik moderner Dialektforschung. Erhebung, Aufbereitung und Auswertung von Daten am Beispiel des Oberdeutschen (pp. 445-470). Hildesheim: Olms.

Glück, A., Leonardi, M. M. V., \& Riehl, C. M. (2019). Südtirol. In R. Beyer, \& A. Plewnia (Eds.), Handbuch des Deutschen in West- und Mitteleuropa. Sprachminderheiten und Mehrsprachigkeitskonstellationen (pp. 245-280). Tübingen: Narr.

Gottas, F. (1995). Die Deutschen in Südosteuropa. Von den Ansiedlungen im Mittelalter und im 18. Jahrhundert zur Rückbewegung im 20. Jahrhundert. In G. Grimm, \& K. Zach (Eds.), 
Die Deutschen in Ostmittel- und Südosteuropa. Geschichte - Wirtschaft - Recht - Sprache: Vol. 1 (pp. 13-30). München: Südostdeutsches Kulturwerk.

Gretschel, H.-V. (1984). Südwesterdeutsch. Eine kritische Bilanz. Logos, 4(2), 38-44.

Huck, D., \& Erhart, P. (2019). Das Elsass. In R. Beyer, \& A. Plewnia (Eds.), Handbuch des Deutschen in West- und Mitteleuropa. Sprachminderheiten und Mehrsprachigkeitskonstellationen (pp. 155-182). Tübingen: Narr.

Kaufmann, G. (2004). Eine Gruppe - zwei Geschichten - eine Sprache. Zeitschrift für Dialektologie und Linguistik, 71, 257-306.

Kloss, H. (1978). Die Entwicklung neuer germanischer Kultursprachen seit 1800. Düsseldorf: Schwann.

Koehler, A. (2017). Quelques effets du contact de langues et de la pression du français sur les dialectes alsaciens: une première approche (Unpublished master thesis, Université de Strasbourg, France).

Mattheier, K. J. (1996). Methoden der Sprachinselforschung. In H. Goebl, P. Nelde, \& Z. Stary (Eds.), Contact Linguistics. An International Handbook of Contemporary Research: Vol. 1 (pp. 812-819). Berlin, New York: De Gruyter.

Protze, H. (1995). Zur Erforschung deutscher Sprachinseln in Südost- und Osteuropa. Siedlung, Sprache, Geschichte und Wechselwirkungen. In G. Grimm, \& K. Zach (Eds.), Die Deutschen in Ostmittel- und Südosteuropa. Geschichte-Wirtschaft-Recht-Sprache: Vol. 1 (pp. 55-84). München: Südostdeutsches Kulturwerk.

Pusch, C. D. (2010). Old minorities within a language space. In P. Auer, \& J. E. Schmidt (Eds.), Language and Space. An International Handbook of Linguistic Variation: Vol. 1. Theories and Methods (pp. 375-390). Berlin, New York: De Gruyter.

Rabanus, S. (2018). Tedesco, Versione 1. Korpus im Text. Innovatives Publizieren im Umfeld der Korpuslinguistik. Serie A, 13187. Retrieved August 21, 2020, from http://www.kit.gwi.unimuenchen.de/?p=13187\&v=1.

Riehl, C. M. (2010). Discontinuous language spaces (Sprachinseln). In P. Auer, \& J. E. Schmidt (Eds.), Language and Space. An International Handbook of Linguistic Variation: Vol. 1. Theories and Methods (pp. 332-354). Berlin, New York: De Gruyter.

Riehl, C. M. (2014). Sprachkontaktforschung. Eine Einführung. Tübingen: Narr.

Riehl, C. M. (2016). Reliktvarietät, Herkunftssprache, Minderheitensprache und neue Mehrsprachigkeit: Das Barossa-Deutsche als Beispiel für die Dynamik der deutschen Sprache in Übersee. In A. Lenz (Ed.), German Abroad. Perspektiven der Variationslinguistik, Sprachkontaktund Mehrsprachigkeitsforschung (pp. 241-267). Wien: V\&R unipress.

Riehl, C. M. (2018). Australien. In A. Plewnia, \& C. M. Riehl (Eds.), Handbuch der deutschen Sprachminderheiten in Übersee (pp. 9-32). Tübingen: Narr.

Riehl, C. M. (2019). Deutsch in Osteuropa. In J. Herrgen, \& J. E. Schmidt (Eds.), Sprache und Raum - Deutsch. Ein internationales Handbuch der Sprachvariation (pp. 1115-1134). Berlin, Boston: De Gruyter.

Rispail, M., Haas-Heckel, M., \& Atamaniuk, H. (2012). Le Platt lorrain pour les Nuls. Paris: Éditions First.

Rosenberg, P. (2005). Dialect convergence in the German language islands (Sprachinseln). In P. Auer, F. Hinskens, \& P. Kerswill (Eds.), Dialect Change. Convergence and Divergence in European Languages (pp. 221-235). Cambridge: Cambridge University Press.

Rosenberg, P. (2016). Regularität und Irregularität in der Kasusmorphologie deutscher Sprachinselvarietäten (Russland, Brasilien). In A. Bittner, \& K.-M. Köpcke (Eds.), Regularität und Irregularität in Phonologie und Morphologie. Diachron, kontrastiv, typologisch (pp. 177217). Berlin: De Gruyter.

Rosenberg, P. (2018). Lateinamerika. In A. Plewnia, \& C. M. Riehl (Eds.). Handbuch der deutschen Sprachminderheiten in Übersee (pp. 193-264). Tübingen: Narr. 
Siemens, H. (2018). Mennoniten in Übersee. In A. Plewnia, \& C. M. Riehl (Eds.), Handbuch der deutschen Sprachminderheiten in Übersee (pp. 265-293). Tübingen: Narr.

Wiese, H., \& Bracke, Y. (2021). Registerdifferenzierung im Namdeutschen. Informeller und formeller Sprachgebrauch in einer vitalen Sprechergemeinschaft. In C. Földes (Ed.), Kontaktvarietäten des Deutschen im Ausland (pp. 273-293). Tübingen: Narr.

Zimmer, C. (2019). Deutsch als Minderheitensprache in Afrika. In J. Herrgen, \& J. E. Schmidt (Eds.), Sprache und Raum - Deutsch. Ein internationales Handbuch der Sprachvariation (pp. 1176-1190). Berlin, Boston: De Gruyter. 